\title{
Future time perspective and trust in government: The mediation of the consideration of future consequences
}

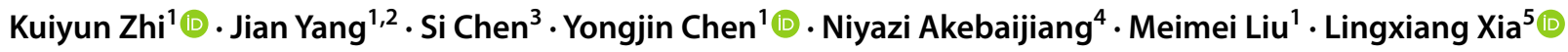

Accepted: 29 September 2021

○) Springer Science+Business Media, LLC, part of Springer Nature 2021

\begin{abstract}
Evidence suggests that college students' future-related feelings and cognition may influence their trust in government. This study aims to explore whether the association between college students' future time perspective and trust in government is mediated through the consideration of future consequences (CFC). A cross-sectional survey was used to collect data from 1,617 college students ( $86.6 \%$ women) aged 17 to 31 years in China via an online questionnaire. The results showed that the students' positive, negative and confused future orientation was positively associated with their trust in government. The consideration of far- future consequences positively mediated the relationship between a positive future orientation and trust in government. These findings support the possibility to evaluate college students' government trust level according to their future orientation, which will improve their positive political participation.
\end{abstract}

Keywords Trust in government $\cdot$ Future time perspective $\cdot$ Consideration of future consequences $\cdot$ College students

\section{Introduction}

College students are in a period of life in which it is essential for them to think about many issues, such as their attitude towards life, survival value and social responsibility (Lattie et al., 2019; Šerek et al., 2018). Life experiences show that they are more likely to plan for their wonderful future life to pursue educational, occupational and social achievement and even lifelong happiness (Chen, 2006). Although they lack social experience, college students are considered to shoulder more social responsibility and play increasingly more

Yongjin Chen

yjchen@cqu.edu.cn

$\triangle$ Lingxiang Xia

xialxn@swu.edu.cn

Kuiyun Zhi

kyzhi@cqu.edu.cn

Jian Yang

yangjian1208@rcu.edu.cn

Si Chen

chensitilla@qq.com

Niyazi Akebaijiang

akebaijiang123@126.com

Meimei Liu

sherryliu0907@163.com important roles in political activities and social governance (Rice et al., 2012). Their political and social behaviors are heavily influenced by their cognition of government (Šerek \& Macek, 2014; Šerek et al., 2018). Trust in government is mostly regarded as the public belief in the trustworthiness of government (Lewicki and Bunker 1995; Hudson, 2006; Kim, 2010; Šerek \& Macek, 2014), which depends on whether the government can observe the rules, serve the public, and satisfy public demands for maintaining social equity and justice over time (Hakhverdian \& Mayne, 2012; Sööt \& Rootalu, 2012, Being regarded as an initial part of adolescents' and

1 School of Public Policy and Administration, Chongqing University, Chongqing City, People's Republic of China

2 School of Public Administration and Policy, Renmin University of China, Beijing City, People's Republic of China

3 School of Business Administration, Chongqing Technology and Business University, Chongqing City, People's Republic of China

4 Aksu Vocational and Technical College, Aksu City, People's Republic of China

5 Faculty of Psychology, Southwest University, Chongqing City, People's Republic of China 
young adults' worldview (Šerek \& Macek, 2014), trust in government develops in college and grows more stable over the course of a person's life (Daas et al., 2019). A lack of trust in government could render individuals more likely to participate in unconventional political activities, such as radical protests and demonstrations, in the future (Šerek \& Macek, 2014; Šerek et al., 2018). Furthermore, college experiences have a significant impact on individuals' future government trust- building (Hooghe et al., 2014; Marien, 2017). Thus, recent studies have increasingly explored the government trust level among college students (Kim, 2019).

Past studies indicated that governments' performance, institutional contexts and values influence trust in government (Chen, 2017; Han et al., 2017; Yang et al., 2021), which are largely based on governmental and environmental characteristics and rarely consider individuals' characteristics. However, college students' cognition of the government contains many future-related factors such as more employment opportunities and the possibility of an income increase (Liu \& Raine, 2015), which directly affect the evaluation of the trustworthiness of government (Fan \& Yan, 2019). Studies have implied that it is easier for positive futureoriented college students to perceive the positive aspects of government and have a higher level of trust in the government (Kim, 2019; Lyu \& Huang, 2016). However, due to early traumatic experiences, individuals who are negative about future events are more likely to perceive a negative or confusing future, which results in a higher risk of distrust in government (Crespo et al., 2013; Gniewosz et al., 2009). The future time perspective involves the thoughts, feelings, and actions related to the future (Chen, 2006; Lyu $\&$ Huang, 2016). The clues above inspired us to explore the relationship between the future time perspective and trust in government.

\section{Future time perspective and trust in government}

The future time perspective (FTP) has been applied to describe feelings about people's future events (Chen, 2002, 2006; Lyu \& Huang, 2016; Zimbardo \& Boyd, 1999). Based on a perceived future event, some people feel positive and hopeful, while some people feel negative and puzzled (Morselli, 2013). For example, work-related events were used to measure college students' future perspective (Cheng, 1997). Some college students perceived positive content, such as "finding an ideal job", but some college students perceived negative content, such as "facing fierce employment competition". Some college students even perceived confusing content, such as "being puzzled about career development". Thus, the FTP reflected feelings about future events from positive to negative and confusing. As previous studies implied, the FTP is suggested to include the following three sub-dimensions based on different feelings: future positive, future negative and future confusion (Lyu \& Huang, 2016). Future positive refers to generally positive, hopeful and happy feelings about future events. Future negative refers to negative, hopeless and pessimistic feelings about future events. Future confusion refers to a confused feeling about future events.

As current Chinese college students have experienced Chinese rapid economic and social development (Liu \& Raine, 2015), most of them are more likely to perceive positive contexts in their daily life and social media such as more disposable income and better job prospects, which makes it easier for them not only to feel that the government is reliable but also to think about a positive future (Chen, 2006). Self-interest theory suggests that those who peruse benefit from the government are more likely to trust the government (Li \& Wu, 2018). High future-positive college students are more likely to perceive the positive aspects from trust in government in the future, such as obtaining rapid responses from the government to personal demands (Fan \& Yan, 2019; Lyu \& Huang, 2016). Meanwhile, as the government is regarded as having made great contributions to China's rapid development (Liu \& Raine, 2015), individuals who pursue their social value may be more likely to work for or coordinate with the government to serve society. Therefore, these students were more likely to take time to build trust with the government and have a higher level of trust in government. Several studies have suggested that a relationship exists between a positive future orientation and trust in government. First, positive future orientated adolescents and young adults reported higher political efficacy (Crocetti et al., 2012). High political efficacy is associated with a high level of trust in government (Eckstein et al., 2013; Manganelli et al., 2014; Šerek et al., 2018). Second, college students with high future expectations were more likely to perceive the value of political engagement and further trust government institutions (Hooghe et al., 2014; Kim, 2019). Third, an open school climate and a high sense of school belonging are beneficial for promoting college students to think about the future positively (Crespo et al., 2013; Goodenow \& Grady, 1993; Hooghe et al., 2014), which results in a high level of trust in the government (Gniewosz et al., 2009). Therefore, we proposed that future positive is positively associated with trust in government (H1a).

Although most college students are more likely to perceive the positive contexts of future events, it may be easier for some of them to perceive negative contexts and uncertainty regarding the future due to early traumatic experiences (Lee et al., 2020). Negative or confused future-oriented college students are more likely to focus on the adverse aspects of future events (Lyu \& Huang, 2016). They are also more likely to lose the control of future time, feel a limited future, and seek immediate satisfaction (Lang \& Carstensen, 2002). Meanwhile, although trust in government has benefits (Fan 
\& Yan, 2019; Kim, 2019), trust- building is a complex process that requires time. Therefore, college students with negative and confused future orientations are more likely to perceive the negative aspects of building trust with the government, such as spending time and energy. It can be asserted that these students are less likely to exert effort to build trust in the government, which indicates a lower level of trust in the government (Lang \& Carstensen, 2002; Lyu $\&$ Huang, 2016). Several studies have implied that a relationship exists among future negative, future confusion and trust in government. First, higher parental control (Crespo et al., 2013) or depression (Ojeda, 2015) could make adolescents and young adults feel negative or confused about the future, which further results in a low level of trust in the government (Glatz \& Dahl, 2014; Gniewosz et al., 2009). Second, interpersonal problems and low optimism render adolescents negative about the future, further rendering them less likely to trust in government institutions (Šerek et al., 2018). Thus, it could be hypothesized that future negative and future confusion are negatively associated with trust in government (H1b).

\section{Consideration of future consequences as a mediator}

Socioemotional selectivity theory advocates that individuals select goals in accordance with their feelings that are based on the cognition of the future as being limited or open-ended (Lang \& Carstensen, 2002). For instance, most younger people such as college students are more likely to have an openended future view and are thus more likely to have goals related to self-benefits or self-realization in society such as acquiring long-term benefits or serving society (Chen, 2006). However, due to terrible past experiences, some college students may perceive limited future time like older people perceive limited future time (Lang \& Carstensen, 2002). They may feel that long and far away future goals are out of reach. Therefore, they are more likely to have emotionally meaningful goals that satisfy them immediately (Lyu \& Huang, 2016). According to socioemotional selectivity theory, different feelings about future events were thought to influence near or far future goal setting, which makes college students consider the consequence of futures with different distances (Chen, 2006; Lang \& Carstensen, 2002; Lyu $\&$ Huang, 2016). The consideration of future consequences (CFC) refers to the cognition of distant future consequences (Strathman et al., 1994; Zhao et al., 2018). The mental time of the future can be classified into the near future and the far future (Chen, 2006). Some people are more likely to act to satisfy immediate concerns, while some people are more likely to pursue a sequence of better far future outcomes, such as demand satisfaction and economic benefits, even though immediate happiness may be sacrificed (Joireman et al., 2008). Considering immediate consequences
(CFC-immediate) and future consequences (CFC-future) are the two major components of CFC (Joireman et al., 2008; Zhao et al., 2018). CFC-immediate emphasizes a focus on immediate future consequences, while CFC-future focuses on far future consequences. Typical immediate future consequences include current amusement, emotional eating, and obtaining immediate but lower financial rewards, while far future consequence include acquiring academic achievements, remaining healthy in the far future and obtaining far greater future financial rewards (Joireman et al., 2008; Kauffman \& Husman, 2004; Xu et al., 2018). Compared with the FTP, CFC focuses on distant consequences rather than certain feelings about future events.

Given college students' optimistic future life that involves, for example, a successful such as marriage and career, Chen (2006) advocated that positive future-oriented college students are more likely to set far future goals and that they thus consider far future consequences more frequently. Existing studies have implied that the FTP is correlated with CFC (Ryack, 2012). For instance, once they set the goal of acquiring academic achievement in the future, positive future-oriented students are more likely to work hard and achieve such a goal (Kauffman \& Husman, 2004). According to some literature, positive future-oriented adolescents and young adults plan carefully and relinquish their current enjoyment to maintain a healthy far future status (Xu et al., 2018). The evidence above suggests that future positive may be associated with $\mathrm{CFC}$-future. Some existing studies suggested that a relationship exists between CFCfuture and trust in government. First, the consideration of behavioral long-term effectiveness drives college students to trust in government and engage in political activities (Eckstein et al., 2013). Second, a study based on Chinese citizens aged from 14 to 65 years indicated that the consideration of future financial returns accounted for a high level of trust in local government (Kostka, 2019). Accordingly, it is possible that a positive future orientation can make people consider far future issues, which, in turn, improves the level of trust in government. Thus, we further propose that CFC-future positively mediates the relationship between future positive and trust in government ( $\mathrm{H} 2 \mathrm{a})$.

Negative or confused future-oriented college students lack future control, which makes them less likely than positive future-oriented college students to perceive positive future events and to thus set far future goals like (Chen, 2006; Lyu \& Huang, 2016). Therefore, they may be less likely to work hard to obtain far positive future outcomes and more likely to focus on near future satisfaction (Lang \& Carstensen, 2002). Evidence suggests that negative or confused future oriented teenagers are more likely to indulge in present satisfactions and consume cannabis without thinking about the future consequences (Apostolidis et al., 2006). Future negative or confused adolescent boys were more 
likely to amuse themselves in gambling and were not sensitive to potential future financial risks (Donati et al., 2019). It could be proposed that future negative and future confused may be associated with CFC-immediate. Studies also suggest that a relationship exists between CFC-immediate and trust in government. Although sample studies based on college students are lacking, evidence suggests that those concerned about immediate benefits, such as daily life resources, are less likely to trust in government (Ong \& Han, 2018). Meanwhile, disappointment about the future caused by corruption makes people consider current issues, significantly reducing trust in government (Ciziceno \& Travaglino, 2018). In summary, it is possible that negative and confused future orientations could make people think about immediate issues, which, in turn, reduces the level of trust in government. Therefore, $\mathrm{CFC}$-immediate may positively mediate the relationship among future negative, future confusion and trust in government $(\mathrm{H} 2 \mathrm{~b})$.

\section{Methods}

\section{Participants and procedures}

We recruited students from two departments of a college in western China to complete a survey. The students were told that they may participate in a survey about college students' future orientations and government-related attitudes. After they read the online consent form, they could choose whether to participate in this survey. The participants were told that this survey would not reveal their personal information, participation was completely voluntary, and joining the survey would authorize researchers to use their data. If the participants were willing to participate in the survey, then they were given a link to this survey. Online questionnaires were sent to them via a website developed by Tencent Company (https://wj.qq.com/). On this platform, the survey data were only accessible to the researcher, which referred to the team members of this project. The participants were instructed to complete the online surveys, including the FTP scale, the CFC scale, and the trust scales. This research was approved by the School of Public Affairs, Chongqing University, China.

In total, 1717 answers were gathered in our study. Questionnaires that were completed too quickly or had invalid answers to many questions were not included in the study. The linear interpolation method was applied to provide missing values. Finally, 1617 valid questionnaires were gathered. Participants aged from 17 to 31 years (average age was 20.12 years) were included in our study. In total, 609 participants were from urban regions (37.7\%), and 1008 participants were from rural regions $(62.3 \%)$.
In total, 1400 participants were women (86.6\%), and 217 participants were men (13.4\%).

This study used Harman's single factor test to examine the potential effects of common method bias. All scale items were subjected to an exploratory factor analysis to determine whether a single factor emerged. The results indicated three factors with three values greater than 1.0, and the first factor accounted for $38.95 \%$ of the accumulation contribution rate $(<40 \%)$. Thus, common method biases were not a problem in this study.

\section{Instruments}

\section{Dependent variables}

This study used the trust in government scale based on the definition. The exploratory factor analysis revealed 17 items loading $>0.70$. Of these, 7 items describe governmental rules (e.g., "I think the government will try to make good on its promises to the public"); 6 items describe the government's ability (e.g., "The government generally does not back out"); and 4 items describe government outcomes in maintaining social equality and justice (e.g., "I think the government will act fairly and not only protect the interests of a few individuals"). A 5-point Likert scale was used to rate the items $(1=$ strongly disagree, $5=$ strongly agree). Similar items were applied in an Asian Barometer Survey (e.g., "Rich and poor people are treated equally by the government" and "The government provides people with quality public services"). The mean of the 17 items was used as the score of the trust in government scale. A higher score indicates a higher level of trust in government. The Cronbach's $\alpha$ coefficient of this scale was 0.84 (the scale is reported in Appendix 1). The confirmatory factor analysis indexes provided an adequate fit of the construct validity of the trust in government scale $\left(\chi^{2}=328.33, d f=89, \chi^{2} / d f=3.69, \mathrm{CFI}=0.966\right.$, $\mathrm{TLI}=0.948, \mathrm{SRMR}=0.100, \mathrm{RMSEA}=0.071)$.

\section{Independent variables}

This study applied a modified future time perspective scale based on a previous study (Lyu \& Huang, 2016). Given the importance of work in college students' future daily life, we use future work-related events, such as career development, to measure feelings towards specific future events in a scare design (Cheng, 1997). This scale consists of 16 items and three subscales named future positive, future negative and future confusion. Future positive includes 6 items (e.g., "I feel hopeful when I think I can find a good job in the future"); future negative includes 7 items (e.g., "I feel sad when I think about the employment pressure after 
graduation"); and future confusion includes 3 items (e.g., "I feel confused because I don't know how to achieve my career goals"). The responses range from strongly disagree (1) to strongly agree (5). A higher score indicates a higher level of FTP. The sums of the three dimensions were the score of the FTP. In Lyu and Huang's study, the Cronbach's $\alpha$ coefficients of future positive, future negative, future confusion and the total scale were $0.83,0.88,0.76$ and 0.90 , respectively. In this study, the Cronbach's $\alpha$ coefficients of future positive, future negative, future confusion and the total scale were $0.91,0.91,0.86$ and 0.91 , respectively. The confirmatory factor analysis indexes provided an adequate fit of the construct validity of the FTP scale $\left(\chi^{2}=465.64, d f=96, \chi^{2} / d f=4.85\right.$, $\mathrm{CFI}=0.980, \mathrm{TLI}=0.970, \mathrm{SRMR}=0.036$, RMSEA $=0.049$ ). The Chinese version of the scale is provided in Appendix 1.

\section{Mediator}

The CFC scale has been used in previous studies (Joireman et al., 2008; Zhao et al., 2018). CFC-immediate and CFC-future are the two sub-dimensions of the scale. CFCimmediate includes 7 items (e.g., "I only act to satisfy immediate concerns and believe that the future will take care of itself"), and CFC-future includes 5 items (e.g., "I consider how things may be in the future and try to influence those things with my day-to-day behavior"). The responses were provided on a 5-point Likert scale ( $1=$ strongly disagree, $5=$ strongly agree). A higher score indicates a higher level of CFC. The sum of the two dimensions was the CFC score. In this study, the Cronbach's $\alpha$ coefficients of CFC-immediate, CFC-future and the whole scale were $0.89,0.89$ and 0.84 , respectively. The confirmatory factor analysis indexes provided an adequate fit of the construct validity of the FTP scale $\left(\chi^{2}=339.93, d f=47, \chi^{2} / d f=7.23, \mathrm{CFI}=0.972\right.$, $\mathrm{TLI}=0.960, \mathrm{SRMR}=0.059, \mathrm{RMSEA}=0.062$ ).

\section{Control variables}

Given that age, gender and household location have been reported to influence trust in government (Zhao et al., 2019), we controlled for these variables in the regression analysis. Age was the actual age, while household location and gender were coded as dummy variables $(0=$ woman and $1=$ man for gender; $0=$ rural and $1=$ urban for region)

\section{Data analysis}

The data were analyzed by Mplus 7.4. First, we reported the correlation coefficients of the study variables. Then, this study established path analysis to examine the relationship between the FTP and trust in government and the mediator of CFC. The coefficients and indirect effects were reported.

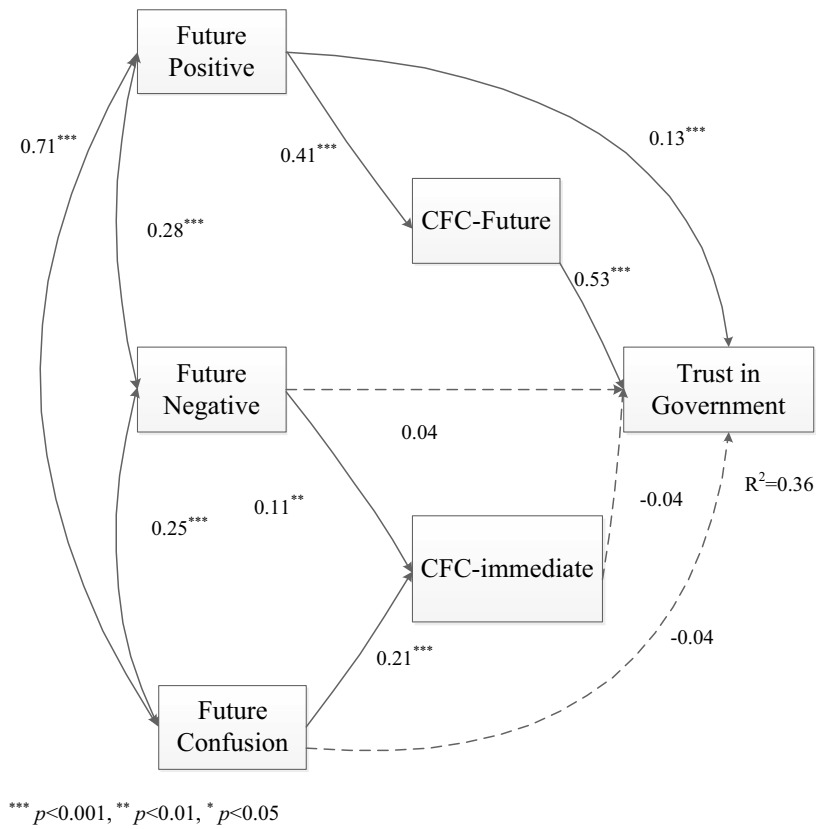

Graph. 1 Regression results of trust in government. ${ }^{* * *} p<0.001,{ }^{* *}$ $p<0.01,{ }^{*} p<0.05$
Table 1 Means, standard deviations and correlation coefficients

\begin{tabular}{llllllllll}
\hline Variable & Mean & $S D$ & 1 & 2 & 3 & 4 & 5 & 6 & 7 \\
\hline 1. Future negative & 3.02 & 0.89 & & & & & & & \\
2. Future positive & 3.65 & 0.91 & $0.29^{* *}$ & & & & & & \\
3. Future confusion & 2.94 & 0.96 & $0.73^{* *}$ & $0.25^{* *}$ & & & & & \\
4. FTP & 3.24 & 0.71 & $0.87^{* *}$ & $0.70^{* *}$ & $0.77^{* *}$ & & & & \\
5. CFC-immediate & 2.62 & 0.91 & $0.25^{* *}$ & $0.07^{* *}$ & $0.28^{* *}$ & $0.24^{* *}$ & & & \\
6. CFC-future & 3.72 & 0.93 & $0.10^{* *}$ & $0.40^{* *}$ & $0.09^{* *}$ & $0.27^{* *}$ & $0.11^{* *}$ & & \\
7. CFC & 3.07 & 0.69 & $0.25^{* *}$ & $0.28^{* *}$ & $0.26^{* *}$ & $0.33^{* *}$ & $0.83^{* *}$ & $0.65^{* *}$ & \\
8. Trust in government & 3.97 & 0.98 & $0.08^{* *}$ & $0.33^{* *}$ & $0.06^{*}$ & $0.22^{* *}$ & 0.05 & $0.59^{* *}$ & $0.37^{* *}$ \\
\hline
\end{tabular}

${ }^{* * * *} p<0.001,{ }^{* * *} p<0.01,{ }^{*} p<0.05$. 


\section{Results}

Table 1 displays the correlations among all study variables. Future positive was moderately associated with trust in government $(r=0.33, p<0.01)$. However, both future negative and future confusion were weakly associated with trust in government ( $r=0.08, p<0.01$ for future negative, $r=0.06$, $p<0.05$ for future confusion). Future positive was moderately associated with CFC-future $(r=0.40, p<0.01)$. In contrast, both future negative and future confusion were moderately associated with CFC-immediate $(r=0.25, p<0.01$ and $r=0.28, p<0.01$, respectively). CFC-future was strongly associated with trust in government $(r=0.59, p<0.01)$. However, the association between CFC-immediate and trust in government was not statistically significant.

Graph. 1 shows the path analysis results. After controlling for the college students' age, gender, and household location, future positive positively predicted CFC-future and trust in government. CFC-future positively predicted trust in government. A significant indirect effect supported that CFC-future positively moderated the relationship between future positive and trust in government $(\beta=0.23, p<0.001)$. $\mathrm{H} 1 \mathrm{a}$ and $\mathrm{H} 2 \mathrm{a}$ were supported. However, future negative and future confusion did not significantly predict trust in government. H1b was not supported. Although future negative and future confusion positively predicted CFC-immediate, the associations between CFC-immediate and trust in government were not statistically significant. Thus, $\mathrm{H} 2 \mathrm{~b}$ was also not supported.

\section{Discussion}

The positive relationships between future positive and trust in government were in accordance with the clues of previous studies, namely, that positive future orientation predicts political engagement and political efficacy (Crocetti et al., 2012; Hooghe et al., 2014; Šerek et al., 2018). Goal-setting of the future time perspective can be applied to explain the study results (Chen, 2006). In general, college students are in a period during which their outlook on life and values are in the phase of formation and stabilization. Given that most of them have an open-ended future view (Chen, 2006; Lang $\&$ Carstensen, 2002), they are likely to set positive future goals such as acquiring long-term benefits and contributing to society, and they are more likely to experience the positive feelings of such future goals (Chen, 2006). Positive future goals enable them to benefit their future development and consider far future issues. Given the government is thought to contribute largely to the rapid economic and social development (Liu \& Raine, 2015), colleges are more likely to positively perceive trust in government (Fan \& Yan, 2019).
Based on Chen's study in 2006, they might then set relevant far future goals such as "join or cooperate with the government to serve society". Relevant goals may push them to interact with the government, and they are more likely to trust the government.

Although a path analysis does not support the significant relationship, the weak positive relationship between negative or confused future orientations and trust in government, as shown in Table 1, was not consistent with our original assumption, and the previous study did not suggest that the negative or confused future orientation reduces trust in government (Šerek et al., 2018). In China, traditional values advocate that citizens should trust the government, follow the government's decisions and allow the government to manage and allocate resources to ensure that efficiency is promoted (Ma \& Wang, 2014). As previously mentioned, college students are an important group of citizens who participate in politics (Lattie et al., 2019; Šerek et al., 2018). Given the Chinese government's good performance in maintaining social and economic development in recent decades, it can be proposed that negative or confused future-oriented college students may follow traditional values and expect the Chinese government to provide more future development-related opportunities and improve their future life quality (Liu \& Raine, 2015). Therefore, the positive relationship between negative or confused future orientations and trust in government could be established in Chinese college students unlike the study in other culture contexts. However, given that path analysis did not support the significant relationship between negative or confused future orientations and trust in government, further examinations need to be conducted in the future.

We find that a positive future orientation may drive college students to be more likely to consider far future consequences and trust in government. This might be helpful for improving college students' positive political and social affairs participation, which benefits their future development. Current college students are expected to contribute more to political issues and society (Rice et al., 2012). Meanwhile, a high level of trust in the government is suggested to stimulate conventional political participation (Šerek \& Macek, 2014; Šerek et al., 2018), which makes college students acquire political knowledge and skills through active participation in social and political affairs (Hooghe et al., 2014; Marien, 2017). By evaluating college students' future orientation, the study results suggest the possibility of encouraging college students' government trust level, which promotes their positive political behaviors.

However, we admit that the major limitation of this study is the lack of evidence to support the causal relationship between the FTP and trust in government. Trust in government was used as the dependent variable and the FTP was 
used as the independent variable in a path analysis. The study does not support the causal relationship between the FTP and trust in government. For instance, although we proposed that a positive future orientation may predict trust in government, we do not know whether a high trust in government promotes a positive future orientation. We mentioned previously that studies based on college students implied that a low trust in government may result in unconventional political participation (Šerek \& Macek, 2014; Šerek et al., 2018); this potentially makes college students perceive the authority to be seeking revenge and thus makes them negative or confused about their future development. A high trust in government may improve political efficacy, which causes college students to be more positive about future events (Manganelli et al., 2014). The evidence above implies that a high trust in government may predict positive future orientation, and a low trust in government may predict a negative or confused future orientation. This study focuses on the factors of trust in government rather than its consequences; therefore, we do not further discuss the potential influence of trust in government on the FTP. Meanwhile, the crosssectional study does not examine the causal relationship. Future studies that obtain longitudinal data to analyze the causal relationship between the FTP and trust in government would be valuable.

This study was limited in several aspects. First, there were significantly more females than males in the sample. The $\mathrm{R}^{2}$ of some tested models was not very high. These limitations may challenge the robustness of this study. Thus, future studies should include samples with balanced participant characteristics and additional data sources (e.g., students from different schools) to validate the results. Second, we used Chinese college students as the participants and fully considered the Chinese context. However, it is an open question whether our conclusions can be supported in other countries with non-college student samples. Therefore, the conclusions require further examination. Finally, we used the association between potential future benefits and trust to demonstrate the meaning of trust; however, many relevant factors, such as culture, may challenge our hypothesis under certain circumstances. For example, considering interpersonal relationships in China, guanxi, which has been used to describe the web of connections used to secure favors among different individuals, should be emphasized. Building guanxi also introduces future benefits even more than trust in China. In this study, we focused on commonality rather than specificity. Thus, future studies that investigate the relationship between future-related time perspectives and trust in other countries should consider culture if specificity is emphasized.

\section{Appendix 1}

Future Time Perspective Scale.

1. I feel sad when I think about the employment pressure after graduation.

2. I feel worried when I think that I might not find the job I want in the future.

3. I feel anxious when thinking about the competition I will face in the job market.

4. I feel anxious when thinking about the great pressure of working in the future.

5. I feel sad that I will not be able to achieve my career goals in the next ten years.

6. I feel frustrated when thinking that I might not be appreciated for years.

7. I feel annoyed when thinking that I might get tired of my job after working for years.

8. I feel hopeful when I think I can find a good job in the future.

9. I feel excited when thinking about the opportunity I'll have after I start working.

10. I feel warm when I think I'll make new friends after I start working.

11. I feel happy when I think that my career will develop very well.

12. I feel happy when I think about the sound development of my position.

13. I feel happy when thinking that I will be appreciated by my superiors in the future.

14. I feel dazed because I don't know what kind of work pressure I'm going to face.

15. I feel confused when I think about the uncertainty of my future career.

16. I feel confused because I don't know how to achieve my career goals.

Trust in Government Scale.

1. I think the government will try to make good on its promises to the public.

2. I am glad that the government can safeguard the public's interests.

3. I don't think the government's style of doing things is confusing and perplexing.

4. Regardless of whether the social environment changes, the government will not change its rules of conduct.

5. I feel at ease that the government is consistent in its work.

6. I think those who work for the government are willing to work hard.

7. The government generally does not back out. 
8. I think those who work for the government will stick to their work.

9. I think the government has the ability to do things.

10. I think it is reassuring and comfortable to delegate to the government.

11. I think the government will act fairly and not only protect the interests of a few individuals.

12. I don't think the government will harm the interests of vulnerable groups.

13. I don't think individuals in government agencies are going to use their power for personal gain.

14. I am not anxious to communicate with the government regarding matters of self-interest.

15. I think the government will still protect the public's interest in the face of major changes.

16. Even if the social environment changes, I will not be worried about what the government is doing.

17. Even if the social environment changes, I will not worry about the results of what the government does.

Acknowledgements The authors thank the editor and the two reviewers for their comments and contributions, which improved the quality of the manuscript.

Authors' contributions $\mathrm{KZ}$ contributed to conceptualization, design, significant manuscript revisions; JY contributed to the theory development, design, data analysis and the write-up; SC contributed to the writing revision and modification; YC contributed to the conceptualization, design and funding; NA contributed to the data collection; ML contributed to the literature search; and LX contributed to the modification.

Funding This study was supported by the Fundamental Research Funds for the Central Universities (grant number 2019CDJSK01PT04, 2018CDJSK01PT05, 2019CDJSK01PY11); the National Social Science Foundation of China Research Program (grant number 21BSH117, 18BGL209).

\section{Declarations}

Ethics approval Ethical approval for this study was granted by the School of Public Policy and Administration at Chongqing University.

Consent to participate Informed consent was provided by the participants online before they started the investigation.

Conflict of interest The authors declare that they have no conflict of interest.

Open Access This article is licensed under a Creative Commons Attribution 4.0 International License, which permits use, sharing, adaptation, distribution and reproduction in any medium or format, as long as you give appropriate credit to the original author(s) and the source, provide a link to the Creative Commons licence, and indicate if changes were made. The images or other third party material in this article are included in the article's Creative Commons licence, unless indicated otherwise in a credit line to the material. If material is not included in the article's Creative Commons licence and your intended use is not permitted by statutory regulation or exceeds the permitted use, you will need to obtain permission directly from the copyright holder. To view a copy of this licence, visit http://creativecommons.org/licenses/by/4.0/.

\section{References}

Apostolidis, T., Fieulaine, N., \& Soulé, F. (2006). Future time perspective as predictor of cannabis use: Exploring the role of substance perception among French adolescents. Addictive Behaviors, 31(12), 2339-2343.

Chen, D. (2017). Local distrust and regime support: Sources and effects of political trust in China. Political Research Quarterly, 70(2), 314-326.

Chen, Y. (2002). The relationship between future time perspective and life events. Psychological Science, 25(2), 233,232.

Chen, Y. (2006). The research on goal orientation of undergraduate future time perspective, doctorial dissertation. Southwest University.

Cheng, C. (1997). Assessment of major life events for hong kong adolescents: The Chinese adolescent life event scale. American Journal of Community Psychology, 25(1), 17-33.

Ciziceno, M., \& Travaglino, G. A. (2018). Perceived corruption and individuals' life satisfaction: The mediating role of institutional trust. Social Indicators Research, 141(2), 685-701.

Crespo, C., Jose, P. E., Kielpikowski, M., \& Pryor, J. (2013). “On solid ground": Family and school connectedness promotes adolescents' future orientation. Journal of Adolescence, 36(5), 993-1002.

Crocetti, E., Jahromi, P., \& Meeus, W. (2012). Identity and civic engagement in adolescence. Journal of Adolescence, 35(3), 521-532.

Daas, R., Dijkstra, A. B., \& Karsten, S. (2019). Assessing young people's citizenship attitudes using rubrics. Studies in Educational Evaluation, 62, 118-128.

Donati, M. A., Sottili, E., Morsanyi, K., \& Primi, C. (2019). Time perspectives and gambling in adolescent boys: Differential effects of present- and future-orientation. Journal of Gambling Studies, $35(1), 107-124$

Eckstein, K., Noack, P., \& Gniewosz, B. (2013). Predictors of intentions to participate in politics and actual political behaviors in young adulthood. International Journal of Behavioral Development, 37(5), 428-435.

Fan, X., \& Yan, F. (2019). The long shadow: Social mobility and political participation in urban China, 2006-2012. Social Science Research, 81, 106-116.

Glatz, T., \& Dahl, V. (2014). The role of family experiences for adolescents' readiness to use and participate in illegal political activity. International Journal of Behavioral Development, 40(1), 11-20.

Gniewosz, B., Noack, P., \& Buhl, M. (2009). Political alienation in adolescence: Associations with parental role models, parenting styles, and classroom climate. International Journal of Behavioral Development, 33(4), 337-346.

Goodenow, C., \& Grady, K. E. (1993). The relationship of school belonging and friends' values to academic motivation among urban adolescent students. The Journal of Experimental Education, 62(1), 60-71.

Hakhverdian, A., \& Mayne, Q. (2012). Institutional trust, education, and corruption: A micro-macro interactive approach. The Journal of Politics, 74(3), 739-750.

Han, Z., Sun, I. Y., \& Hu, R. (2017). Social trust, neighborhood cohesion, and public trust in the police in China. Policing: An International Journal of Police Strategies \& Management, 40(2), 380-394. 
Hooghe, M., Dassonneville, R., \& Marien, S. (2014). The impact of education on the development of political trust: Results from a five-year panel study among late adolescents and young adults in Belgium. Political Studies, 63(1), 123-141.

Hudson, J. (2006). Institutional trust and subjective well-being across the EU. Kyklos, 59(1), 43-62.

Joireman, J., Balliet, D., Sprott, D., Spangenberg, E., \& Schultz, J. (2008). Consideration of future consequences, ego-depletion, and self-control: Support for distinguishing between CFC-immediate and CFC-future sub-scales. Personality and Individual Differences, 45(1), 15-21.

Kauffman, D. F., \& Husman, J. (2004). Effects of time perspective on student motivation: Introduction to a special issue. Educational Psychology Review, 16(1), 1-7.

Kim, H. (2019). A cross-national examination of political trust in adolescence: The effects of adolescents' educational expectations and country's democratic governance. Young, 27(3), 245-278.

Kim, S. (2010). Public trust in government in Japan and South Korea: Does the rise of critical citizens matter? Public Administration Review, 70(5), 801-810.

Kostka, G. (2019). China's social credit systems and public opinion: Explaining high levels of approval. New Media \& Society, 21(7), 1565-1593.

Lattie, E. G., Lipson, S. K., \& Eisenberg, D. (2019). Technology and college student mental health: Challenges and opportunities. Front Psychiatry, 10, 246.

Lang, F. R., \& Carstensen, L. L. (2002). Time counts: Future time perspective, goals, and social relationships. Psychology and Aging, 17(1), 125-139.

Lee, D. B., Hsieh, H. F., Stoddard, S. A., Heinze, J. E., Carter, P. M., Goldstick, J. E., et al. (2020). Longitudinal pathway from violence exposure to firearm carriage among adolescents: The role of future expectation. Journal of Adolescence, 81, 101-113.

Lewicki, R. J., \& Bunker, B. B. (1995). Trust in relationships: A model of trust development and decline. In B. B. Bunker \& J. Z. Rubin (Eds.), Conflict cooperation and justice: Essays inspired by the work of Morton Deutsch (pp. 133-174). San Francisco: Jossey-Bass.

Li, Z., \& Wu, X. (2018). Social policy and political trust: Evidence from the new rural pension scheme in China. The China Quarterly, 235, 644-668.

Liu, H., \& Raine, J. W. (2015). Why is there less public trust in local government than in central government in China? International Journal of Public Administration, 39(4), 258-269.

Lyu, H., \& Huang, X. (2016). Development and validation of future time perspective scale for adolescents and young adults. Time \& Society, 25(3), 533-551.

Ma, D., \& Wang, Z. (2014). Governance Innovations and Citizens' Trust in Local Government: Electoral Impacts in China's Townships. Japanese Journal of Political Science, 15(3), 373-395.

Manganelli, S., Lucidi, F., \& Alivernini, F. (2014). Adolescents' expected civic participation: The role of civic knowledge and efficacy beliefs. Journal of Adolescence, 37(5), 632-641.

Marien, S. (2017). Assessing the role of television, the family, and the school in the development of political trust in adolescence. Social Science Quarterly, 98(3), 818-835.
Morselli, D. (2013). The olive tree effect: Future time perspective when the future is uncertain. Culture \& Psychology, 19(3), 305-322.

Ojeda, C. (2015). Depression and political participation. Social Science Quarterly, 96(5), 1226-1243.

Ong, L. H., \& Han, D. (2018). What drives people to protest in an authoritarian country? Resources and rewards vs risks of protests in urban and rural China. Political Studies, 67(1), 224-248.

Park, I. J., Rie, J., Kim, H. S., \& Park, J. (2018). Effects of a future time perspective-based career intervention on career decisions. Journal of Career Development, 47(1), 96-110.

Rice, L. L., Moffett, K. W., \& Madupalli, R. (2012). Campaign-related social networking and the political participation of college students. Social Science Computer Review, 31(3), 257-279.

Ryack, K. (2012). Evidence that time perspective factors depend on the group: Factor analyses of the CFC and ZTPI scales with professional financial advisors. Personality and Individual Differences, 52(6), 723-727.

Šerek, J., \& Macek, P. (2014). Antecedents of political trust in adolescence: Cognitive abilities and perceptions of parents. Journal of Applied Developmental Psychology, 35(4), 284-293.

Šerek, J., Machackova, H., \& Macek, P. (2018). Who crosses the norms? Predictors of the readiness for non-normative political participation among adolescents. Journal of Adolescence, 62, $18-26$.

Sööt, M. L., \& Rootalu, K. (2012). Institutional trust and opinions of corruption. Public Administration and Development, 32(1), 82-95.

Strathman, A., Gleicher, F., Boninger, D. S., \& Edwards, C. S. (1994). The consideration of future consequences: Weighing immediate and distant outcomes of behavior. Journal of Personality and Social Psychology, 66(4), 742-752.

Xu, Y., Yang, Y., \& Ma, X. (2018). Time perspective, optimistic bias, and self-control among different statuses of university smokers in China: A cross-sectional study. Psychology, Health \& Medicine, 23(9), 1054-1059.

Yang, J., Dong, C., \& Chen, Y. (2021). Government's Economic Performance Fosters Trust in Government in China: Assessing the Moderating Effect of Respect for Authority. Social Indicators Research, 154(2), 545-558.

Zhao, D., Zhao, H., \& Cleary, P. D. (2019). Understanding the determinants of public trust in the health care system in China: An analysis of a cross-sectional survey. Journal of Health Services Research \& Policy, 24(1), 37-43.

Zhao, Y., Wei, J., Chen, Y., \& Xia, L. (2018). Consideration of future consequences (CFC) serves as a buffer against aggression related to psychopathy. PLoS One, 13(9), e0203663.

Zimbardo, P. G., \& Boyd, J. N. (1999). Putting time in perspective: A valid, reliable individual-differences metric. Journal of Personality and Social Psychology, 77(6), 1271-1288.

Publisher's note Springer Nature remains neutral with regard to jurisdictional claims in published maps and institutional affiliations. 